\title{
El diagnóstico comercial: herramienta básica para la gestión empresarial
}

\author{
The Commercial Diagnosis: Basic Tool for Business Management \\ Olivia Jiménez Diez ${ }^{a}$, Ana Leticia Rosado Cervera, ${ }^{b}$ Arlen Cerón Islas ${ }^{c}$
}

\begin{abstract}
:
Companies today face diverse changes that imply modifications from their creation, each of value to the exchange with their environment, which undoubtedly requires a modification of the operation and management profile; what undoubtedly requires developing a strategy that allows the improvement and monitoring of organizational changes, which is called a commercial diagnosis.

Keywords:

Commercial diagnosis, management, strategy

Resumen:

Las empresas enfrentan hoy día cambios diversos que implican las modificaciones desde su creación, cada de valor hasta el intercambio con su entorno, lo que sin duda requiere una modificación del perfil de operación y gestión; lo que requiere sin duda desarrollar una estrategia que permita la mejora y monitoreo de los cambios organizacionales, lo que se denomina un diagnostico comercial.
\end{abstract}

Palabras Clave:

Diagnóstico commercial, gestiòn, estrategía.

\section{Introducción}

Hoy en día los conceptos de productividad, eficiencia y calidad ocupan un lugar central dentro de la esfera empresarial y de los centros de investigación. Esto debido a que se ha tomado conciencia de la necesidad de lograr organizaciones que ofrezcan mayores posibilidades de desarrollo a sus miembros, y también porque se requiere que las organizaciones tengan formas más adecuadas de configuración que permitan mejores niveles de productividad y calidad dentro de un clima más humano.

\section{Diagnóstico commercial}

Las empresas enfrentan diversos cambios, desde modificaciones en la cadena de suministros, de producción, de diversificación, hasta cambios en las regulaciones y normas, entre otros; estos tienen incidencia en la empresa modificando su perfil de operación y gestión. En algunos casos, los cambios son grandes eventos y en otros, son cambios pequeños pero que sumados pueden marcar la diferencia al momento de evaluar un antes y un actual en el desempeño empresarial.

Estos cambios pueden, provocar que la organización quede fuera de contexto al no adecuarse a la demanda, y por ende no poder hacerle frente a la competencia, lo cual puede poner en riesgo la supervivencia de la empresa misma.

Por ello es necesario conocer objetivamente lo que está sucediendo, y poder diseñar e implementar un plan de acción que permita revertir la situación, antes de que sea demasiado tarde

Para ello una estrategia útil es el desarrollo organizacional (D.O.), proceso de mejora planeado y monitoreado por medio de evaluaciones integrales periódicas que dan como resultado la supervivencia y mejora integral en las empresas.

EI D.O. es una compleja estrategia que pretende cambiar las creencias, actitudes, valores y estructura de las

\footnotetext{
a, Profesor de tiempo completo, Universidad Autónoma de Yucatán, Facultad de Ciencias Contables y Administrativas, Licenciatura en Administración; https://orcidorg/0000-0002-4514-5241; Email: jdiez@correo.uady.mx

b Maestro por asignatura, Universidad Autónoma de Yucatán, Facultad de Ciencias Contables y Administrativas, Licenciatura en Administración; https://orcid.org/ 0000-0001-7360-7522, Email: ana.rosadocervera@gmail.com

c Autor de Correspondencia. Profesor investigador, Universidad Autónoma del Estado de Hidalgo, Instituto de Ciencias Económico Administrativas, Área Académica de Mercadotecnia, https://orcid.org/0000-0003-3982-5053, Email: arlenc@uaeh.edu.mx
} 
organizaciones para que puedan adaptarse mejor a las nuevas tecnologías, mercados y retos. [1]

Por tanto, el D.O. no es solo una acción realizada para mejorar una organización, no es la función de capacitación de la organización, es un tipo particular de cambio en un proceso determinado para lograr un tipo de resultado final especifico. EI D.O. puede implicar intervenciones en la organización, ya sea en los procesos, en la utilización de habilidades personales, así como la reflexión, la mejora de sistemas, la planificación y el auto-análisis.

La teoría del cambio planeado describe las diferentes etapas por las cuales se transita para lograr introducir el cambio planeado en las organizaciones Algunos de estos modelos se pueden apreciar en la tabla 1, la cual muestra las características comunes que guardan entre sí.

\begin{tabular}{|c|c|c|c|c|}
\hline Características & Modelo de Lewin & $\begin{array}{l}\text { Modelo de Lippitt, } \\
\text { Watson y Wesley }\end{array}$ & $\begin{array}{l}\text { Modelo } \\
\text { Investigación- } \\
\text { acción }\end{array}$ & $\begin{array}{l}\text { Modelo de } \\
\text { Faria Mello }\end{array}$ \\
\hline $\begin{array}{l}\text { Descripción de } \\
\text { fases de cambio }\end{array}$ & $\mathrm{x}$ & $\mathrm{x}$ & $\mathrm{x}$ & $\mathrm{x}$ \\
\hline \multirow{2}{*}{$\begin{array}{l}\text { Cada fase de } \\
\text { cambio le antecede } \\
\text { una fase preliminar }\end{array}$} & $\mathrm{x}$ & $\mathrm{x}$ & $x$ & $x$ \\
\hline & $\begin{array}{l}\text { Descongelamient } \\
\text { o }\end{array}$ & Diagnóstico & $\begin{array}{l}\text { Planeación de la } \\
\text { acción }\end{array}$ & Diagnóstico \\
\hline $\begin{array}{ll}\text { A cada fase } & \text { de } \\
\text { cambio } & \text { le } \\
\text { sobreviene } & \text { un } \\
\text { estado terminal } & \end{array}$ & Recongelamiento & Evaluación & Evaluación & $\begin{array}{l}\text { Planeación de } \\
\text { intervenciones }\end{array}$ \\
\hline $\begin{array}{l}\text { Se enfoca en el } \\
\text { proceso general o } \\
\text { cambio planeado }\end{array}$ & $\mathrm{x}$ & - & - & - \\
\hline $\begin{array}{l}\text { Se enfoca en las } \\
\text { actividades } \\
\text { específicas del D.O. }\end{array}$ & - & $\mathrm{x}$ & $\mathrm{x}$ & $x$ \\
\hline $\begin{array}{l}\text { Descripción de un } \\
\text { proceso de cambio } \\
\text { planeado }\end{array}$ & $\mathrm{x}$ & - & - & - \\
\hline $\begin{array}{ll}\text { Explicación de las } & \text { lades del } \\
\text { actividades } & \text { de } \\
\text { cambio planeado }\end{array}$ & - & $\mathrm{x}$ & $\mathrm{x}$ & $x$ \\
\hline $\begin{array}{lr}\text { Enfatiza } & \text { la } \\
\text { aplicación del } \\
\text { conocimiento de la } \\
\text { ciencia } & \text { del } \\
\text { comportamiento } & \end{array}$ & - & $\mathrm{x}$ & $\mathrm{x}$ & $\mathrm{x}$ \\
\hline $\begin{array}{l}\text { Involucra el uso de } \\
\text { grupos }\end{array}$ & - & $\mathrm{x}$ & $\mathrm{x}$ & $\mathrm{x}$ \\
\hline $\begin{array}{l}\text { Reconoce la } \\
\text { interacción entre un } \\
\text { consultor y una } \\
\text { organización }\end{array}$ & - & $x$ & $\mathrm{x}$ & $x$ \\
\hline $\begin{array}{lll}\begin{array}{l}\text { Enfatizan en } \\
\text { realización } \\
\text { intervenciones }\end{array} & \text { de } \\
\end{array}$ & - & $\mathrm{x}$ & $\mathrm{x}$ & $\mathrm{x}$ \\
\hline $\begin{array}{l}\text { Enfatiza en la } \\
\text { solución de futuros } \\
\text { problemas }\end{array}$ & - & - & $\mathrm{x}$ & $\mathrm{x}$ \\
\hline $\begin{array}{l}\text { Son } \\
\text { cíclicos }\end{array}$ & $x$ & $x$ & $x$ & $x$ \\
\hline $\begin{array}{l}\text { Conduce a la } \\
\text { exploración } \\
\text { constante }\end{array}$ & $x$ & $x$ & $\mathrm{x}$ & $\mathrm{x}$ \\
\hline
\end{tabular}

Tabla 1. Comparación de modelos de D.O.

Fuente: Guízar, 1998; Fleitman, 2001.

El D.O. se concentra en generar cambios en el entorno laboral que ayudan a una organización a adaptarse mejor a su ambiente actual o mejoran su adecuación a los ambientes futuros esperados.
Aun cuando la evaluación integral es un proceso poco usado sobre todo en el área comercial, su aplicación puede marcar una gran diferencia en la permanencia y crecimiento de la empresa.

De los cuatro modelos señalados, dos de ellos identifican al diagnóstico como una herramienta básica para la detección de problemáticas. Dentro de una organización, el diagnóstico es importante ya que nos ayuda a observar situaciones que de otra manera no se ven, dado el estado de inmersión en el día a día, con lo cual las contingencias se vuelven comunes y es muy sencillo acostumbrase a coexistir con estas situaciones que no deberían estar presente.[2]

Un diagnóstico implica la valoración de una situación y toda evaluación corresponde a un proceso que permite evaluar acciones y/o resultados en relación con ciertos objetivos que se establecieron. El objetivo del diagnóstico comercial es determinar la capacidad de la organización para avanzar y permanecer en su mercado a través de la aplicación de nuevas formas de organización y comercialización con innovación estratégica. Su importancia radica en:

1. Contar con toda la información existente para lograr un mayor grado de certeza en la definición del plan comercial de la organización.

2. Determinar las fortalezas y debilidades propias de la organización y del producto en relación a las oportunidades y amenazas existentes, que permite el diseño de una buena estrategia competitiva.

3. Disminuir la incertidumbre en relación a la realidad actual de la empresa.

Al realizar un diagnóstico comercial se deben evaluar seis principales componentes de la organización comercial de la empresa (Ver fig.1):[3]

1. Entorno Comercial

2. Estrategia comercial

3. Organización comercial

4. Sistemas comerciales

5. Productividad comercial

6. Funciones comerciales 


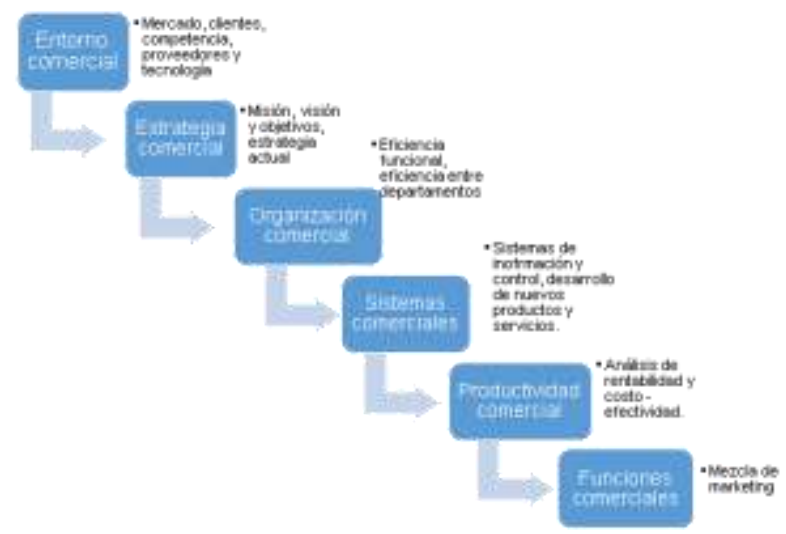

Figura 1. El diagnóstico comercial.

Fuente: Fleitman, 2001.

Particularmente en el diagnóstico de la gestión comercial de una empresa, se busca determinar la posición de la organización en el mercado en que está presente y su grado de competitividad, rentabilidad y posibilidades futuras, así como la calidad de sus productos y servicios.

[4]

Por lo anterior, se puede afirmar que el diagnóstico comercial es una herramienta indispensable para lograr la competitividad, ya que ayuda a definir y comunicar lo referente a la ventaja competitiva, el plan comercial, y la medición y control de indicadores críticos para monitorear el buen funcionamiento del área comercial, que trae consigo la permanencia de la empresa en el mercado y la fidelización de los clientes.

\section{Referencias}

[1] Warren, B. (1996). Organizational revitalization. California Management Review. October, 1966; 9(1): 51-60.

[2] Guízar, R. (1998) Desarrollo Organizacional. Principios y aplicaciones. México: McGraw Hill. 1998.

[3] Ferrance, E. Actión Research. LA. Brown University. Richmond. 2000.

[4] Fleitman, J. (2001) Desarrollo organizacional. Enfoque integral. Limusa, México. 2001. 